Selection 2 (2001) 1-2, 119-131

Available online at http://www.akkrt.hu

\title{
Evolutionary Branching and the Evolution of Anisogamy
}

\author{
N. MAIRE ${ }^{1}$ M. ACKERMANN ${ }^{1}$ and M. DOEBELI ${ }^{2 *}$ \\ ${ }^{1}$ Zoology Institute, University of Basel, Basel, Switzerland, \\ ${ }^{2}$ Department of Zoology, University of British Columbia, Vancouver, B.C., Canada
}

(Received: 17 May 2001,

Accepted: 20 August 2001)

\begin{abstract}
Populations of most sexual species are anisogamous, i.e. they consist of two types of individuals producing gametes of different size. The evolution of anisogamy is usually explained with models that either rely on mutations with large effects or are based on populations with pre-existing mating types. Here we present a model for the evolution of anisogamy that does not rely on either of those assumptions. We used the theory of adaptive dynamics to study the evolution of gamete size allowing arbitrarily small mutations in a population without mating types. As in previous models, we assumed that the survival of a zygote depends on its size, and therefore on the sum of the sizes of the gametes that formed it, and we altered the form of this relationship to investigate a broad range of ecological conditions. We found that the emergence of gamete size dimorphism is a frequent and general evolutionary outcome of our models. Using individual-based models we further show that gamete size dimorphism frequently leads to anisogamous populations consisting of two types of genotypes that differ in the size of the gametes they produce.
\end{abstract}

Keywords: Isogamy, anisogamy, genetic model, evolutionary branching, adaptive dynamics, tradeoff, allocation, disruptive selection, fitness minimum

\section{Introduction}

Most sexual eukaryotic species are anisogamous, that is, they contain two types of genotypes that differ in the size of the gametes they produce. Anisogamy is most likely a derived state that has evolved from ancestors with isogamous reproduction and is generally believed to be the origin of all further sexual dimorphism. Understanding the selective forces leading to the evolution of anisogamy is therefore an important issue in evolutionary biology that has been addressed by a number of studies during the past three decades. Our current understanding of the evolution of anisogamy de-

* Corresponding author: M. Doebeli, Department of Zoology, University of British Columbia, 6270 University Boulevard, Vancouver B.C., Canada V6T 1Z4, Tel (604) 822-3326; Fax (604) 822-2416; E-mail: doebeli@zoology.ubc.ca rives mainly from a model of Parker et al. (1972) [see also Bell (1978, 1982); Maynard Smith (1978), Charlesworth (1978); Hoekstra (1980, 1987); Bulmer (1994)], which is based on two assumptions. The first assumption is that the survival of a zygote depends on its size, where zygote size is determined by the size of the two gametes that fuse to form the zygote. The second assumption is that the amount of resources that an individual allocates to reproduction is constant, so that gamete size trades off with the number of gametes produced. Two opposing forces therefore impinge on the evolution of gamete size: Production of a large number of small gametes increases the number of fusions, whereas a smaller number of larger gametes increases the survival of the resulting zygotes. Moreover, the relative importance of these forces is frequency-dependent: The more large gametes are present, the more advantageous it is to 
produce many small gametes. Parker et al. (1972) showed that this frequency-dependence can lead to disruptive selection on gamete size. In their model alleles encoding for large and small gametes are favoured over alleles encoding for gametes of intermediate size if the range of gamete size is large enough (and based on genetic differences). The genetic variability required for this process to work can only be generated by mutations with large effects, as selection on gamete size in their model is always stabilizing if only sufficiently small mutations are considered. How much genetic variance is needed to induce the evolution of anisogamy depends on the exact form of the relationship between zygote size and zygote survival (Charlesworth, 1978). We note that once a protected polymorphism of alleles coding for large and small gametes has been established, further divergence of this size dimorphism does not require mutations to exceed a minimal effect (Hoekstram, 1980). The initial dimorphism, however, can only be established if the size of the mutations exceeds a critical value.

Although it is conceivable that mutations affecting the timing and number of the cell divisions preceding gamete formation can have a drastic effect on gamete size (Parker et al., 1972), mutations with a large phenotypic effect are often assumed to be deleterious. It is therefore important to investigate whether anisogamy can, in principle, evolve with infinitesimally small mutations, which is the main goal of the present paper. We approach this question by analysing the basic model of Parker et al. (1972) in the framework of adaptive dynamics (Geritz et al., 1998). Adaptive dynamics provides a tool for studying evolutionary dynamics by analysing the invasion success of rare mutants into monomorphic resident populations. One paradigmatic pattern of special interest in adaptive dynamics is evolutionary branching (Geritz et al., 1998), during which an initially monomorphic population splits into two (or more) distinct clusters. This evolutionary split occurs gradually and is a consequence of prior evolution towards a fitness minimum. In particular, no lower limit for the size of mutational effects is required for the diverging polymorphism to develop. In evolutionary models for gamete size evolutionary branching in the allele determining gamete size would lead to the production of genotypes that differ in the size of the gam- etes they produce, which is a prerequisite for the evolution of anisogamy. Therefore, we study the adaptive dynamics in the space of alleles affecting gamete size and the conditions that lead to evolutionary branching under the assumptions that zygote survival depends on size and that the reproductive effort is constant. In contrast to previous models we will explore different functions for the relationship between the size of a zygote and its survival, and we will not rely on the assumption of an initial variance in gamete size. Instead, in adaptive dynamics one starts from monomorphic populations and then analyses the fate of mutant alleles that differ only slightly from the resident allele. This approach allows us to develop an analytical theory for the evolutionary dynamics of gamete size and to identify the conditions under which this dynamics converges to a fitness minimum in allele space, and hence to an evolutionary branching point. As we will see, these branching points are the potential starting points for the evolution of anisogamy.

Following the analytical treatment, we use individual based computer simulations to test whether the evolutionary branching in allele space indeed leads to the evolution of anisogamy. Anisogamy in natural systems is characterized by the presence of two genotypes that differ in the size of the gametes they produce. Evolutionary branching in allele space will initially give rise to the formation of three different genotypes. We demonstrate that one of these genotypes is often lost in the further course of evolution so that anisogamy based on two different genotypes is attained. Overall, our results suggest that evolutionary branching of gamete size is a plausible mechanism for the evolution of anisogamy.

\section{The model}

Following Parker et al. (1972), we assume that gamete size is determined by the parental genotype, e.g. through resource allocation during gametogenesis in a diplontic life cycle. Accordingly, the size of gametes produced by a diploid parent individual is determined by two alleles $(x, y)$ at one locus. All gametes produced by an $(x, y)$-individual will have size $(x+y) / 2$, but one half of these gametes will carry the $x$-allele, and the other half will 
carry the $y$-allele. For example, in a population that is monomorphic for gamete size allele $x$, so that all diploid parent individuals have genotype $(x, x)$, all gametes will be of size $(x+x) / 2=x$, and of course all gametes will carry the $x$-allele. As a consequence, all zygotes have size $2 x$, because zygote size is simply the sum of the size of the gametes that fuse to form the zygote. Because we always assume a constant total reproductive effort, the number of gametes produced is proportional to the inverse of the size of the gametes produced. For example, the number of gametes produced by an $(x, x)$-individual is proportional to $1 / x$.

As in the classical models of Parker et al. (1972) and Charlesworth (1978) we assume a model with discrete, non-overlapping generations. To set up the model for adaptive dynamics, it is most convenient to keep track of the reproductive output of single gamete size alleles, rather than the reproductive output of diploid individuals (although the latter approach would lead to an equivalent description). Consider a single allele $x$ in a population that is monomorphic for gamete size allele $x$. The focal $x$-allele then occurs in an individual of genotype $(x, x)$. This individual produces gametes of genotype $x$ and size $x$. The number of these gametes is proportional to $1 / x$. Half of these gametes will carry copies of the focal $x$-allele under consideration and they will fuse with other gametes of size $x$ to form zygotes of size $2 x$. These zygotes have probability of survival $\mathrm{K}(2 x)$, and therefore the reproductive output of the focal $x$ allele in a population that is monomorphic for $x$, a quantity that we denote by $M(x, x)$, is

$$
M(x, x)=\frac{K(2 x)}{2 x} .
$$

To study the evolutionary dynamics of the gamete size alleles, we investigate the fate of a rare mutant allele $y$ in a population that is monomorphic for gamete size allele $x$, as is the standard procedure in adaptive dynamics (Geritz et al., 1998). If the mutant allele $y$ is rare, it finds itself exclusively in individuals of genotype $(x, y)$. These $(x, y)$-individuals produce gametes of size $(x+y) / 2$, hence the number of gametes they produce is proportional to $2 /(x+y)$. Half of these gametes will carry the $y$-allele, and, as $y$ is rare, they will fuse with gametes of size $x$ and genotype $x$ to produce zy- gotes of size $(x+(x+y) / 2)$. These zygotes will survive with probability $K(x+(x+y) / 2)$, and hence the reproductive output of a $y$-allele in a resident that is monomorphic for $x$, a quantity that we denote by $M(y, x)$, is proportional to

$M(y, x)=\frac{2 K\left(x+\frac{y+x}{2}\right)}{2(y+x)}=\frac{K\left(x+\frac{y+x}{2}\right)}{x+y}$.

If we assume that population size is constant, which we always do in this paper, the fate of the mutant $y$ is determined by its relative reproductive success compared to the resident allele $x$. Since the mutant is rare, the reproductive output of a resident allele $x$ is still given by $M(x, x)$, eq. (1) above, hence the relative reproductive success of a mutant allele $y$ in a resident that is monomorphic for $x$, a quantity that we denote by $f(y, x)$, is given by

$f(y, x)=\frac{M(y, x)}{M(x, x)}=\frac{K\left(x+\frac{(y+x)}{2}\right)}{K(2 x)} \cdot \frac{2 x}{(y+x)}$.

Note that $f(x, x)=1$, which corresponds to the neutral scenario in which a mutant allele that is identical to the resident on average leaves the same number of offspring as the resident, i.e. one per generation. If the reproductive success of a rare mutant allele $y$ is greater than the success of the resident allele $x$ (i.e. $f(y, x)>1$ ), then $y$ will increase in frequency and invade. In accordance with standard adaptive dynamics theory (Geritz et al., 1998) the direction of the evolutionary dynamics is therefore determined by the fitness gradient

$$
g(x)=\left.\frac{\partial f(y, x)}{\partial y}\right|_{y=x} .
$$

If $g(x)>0$, then alleles coding for larger gametes than the current resident have a higher reproductive output and can therefore invade, hence gamete size will increase evolutionarily. Conversely, if $g(x)<0$ then gamete size will decrease. Both these process are either stopped by external constraints, or if a gamete size $x^{*}$ is reached for which the selection gradient vanishes. Such gamete sizes are called singular points and are of particular interest, because they are potential endpoints of the evolutionary process. A population will evolve towards 
a singular point $x^{*}$ if $g(x)$ is positive for residents $x$ $<x^{*}$ and negative for $x>x^{*}$. In other words, $x^{*}$ is an attractor for the adaptive dynamics given by the fitness gradient if

$$
\left.\frac{d g(x)}{d x}\right|_{x=x^{*}}<0 .
$$

To determine the further course of evolution after the system has reached an evolutionary attractor $x^{*}$ one has to consider the second derivative of the invasion fitness, eq. 3 , at the singular point, i.e. the quantity

$$
\left.\frac{\partial^{2} f\left(y, x^{*}\right)}{\partial^{2} y}\right|_{y=x^{*}} .
$$

According to the sign of this quantity, there are two different kinds of evolutionary attractors $x^{*}$. If

$$
\left.\frac{\partial^{2} f\left(y, x^{*}\right)}{\partial^{2} y}\right|_{y=x^{*}}<0
$$

then no nearby mutant can invade the evolutionary attractor $x^{*}$, and hence $x^{*}$ is an evolutionary stable strategy (ESS). In our model, an attractor $x^{*}$ that is also an ESS would correspond to a population with evolutionarily stable isogamy.

A completely different scenario occurs if

$$
\left.\frac{\partial^{2} f\left(y, x^{*}\right)}{\partial^{2} y}\right|_{y=x^{*}}>0 .
$$

Then the attractor $x^{*}$ actually represents a fitness minimum, and therefore every nearby mutant can invade. In 1-dimensional trait spaces such as the space of allelic effects on gamete size considered here, evolutionarily attracting fitness minima are called evolutionary branching points (Geritz et al., 1998), because evolutionary convergence to the fitness minimum will necessarily be followed by a dimorphic split in trait space (Geritz et al., 1998).

In our model, a population that has evolved to a branching point will experience disruptive selection on gamete size so that mutant alleles coding for both larger and smaller gamete size can invade. As will be described below, this process can potentially lead to anisogamy, i.e. to a population consisting of two distinct types of individuals, one type producing small gametes, the other type producing large gametes.

For any given function for zygote fitness $K(z)$, it is rather easy to calculate the resulting fitness gradient, eq. (4), the evolutionary singular points at which the fitness gradient vanishes, as well as their convergence stability [eq. (5)] and their evolutionary stability [eq. (6)]. In fact, for any $K(z)$ the fitness gradient is obtained from eq. (3) as

$$
g(x)=\left.\frac{\partial f(y, x)}{\partial y}\right|_{y=x}=\frac{K^{\prime}(2 x)}{2 K(2 x)}-\frac{1}{2 x}
$$

where $K^{\prime}(z)$ denotes the derivative of $K(z)$.Thus, evolutionary singular points are the solutions $x^{*}$ of the equation

$$
x^{*}=\frac{K\left(2 x^{*}\right)}{K^{\prime}\left(2 x^{*}\right)} .
$$

These singular points are convergent stable for the adaptive dynamics if

$$
\frac{d g}{d x}\left(x^{*}\right)=\frac{K^{\prime \prime}\left(2 x^{*}\right)}{K\left(2 x^{*}\right)}-\frac{1}{2 x^{* 2}}<0
$$

where $K^{\prime \prime}(z)$ denotes the second derivative of $K(z)$, and where we used eq. (10) to derive the expression for $d g\left(x^{*}\right) / d x$. Finally, to evaluate the evolutionary stability of a singular point we again use eq. (10) to calculate

$$
\left.\frac{\partial^{2} f(y, x)}{\partial y^{2}}\right|_{y=x=x^{*}}=\frac{K^{\prime \prime}\left(2 x^{*}\right)}{4 K\left(2 x^{*}\right)} .
$$

Thus, a singular point $x^{*}$ is evolutionarily unstable if

$$
K^{\prime \prime}\left(2 x^{*}\right)>0 \text {. }
$$

In the sequel we use expressions (9)-(13) to analyze the adaptive dynamics of gamete size for different choices of the zygote fitness function $K(z)$.

Of course, we are mostly interested in the conditions that lead to evolutionary branching, since this is the scenario that can lead to the evolution of anisogamy.

We start with the following function relating zygote size to zygote survival (Fig. 1a), which is a modified version of the function that Parker et al. use in their original model: 

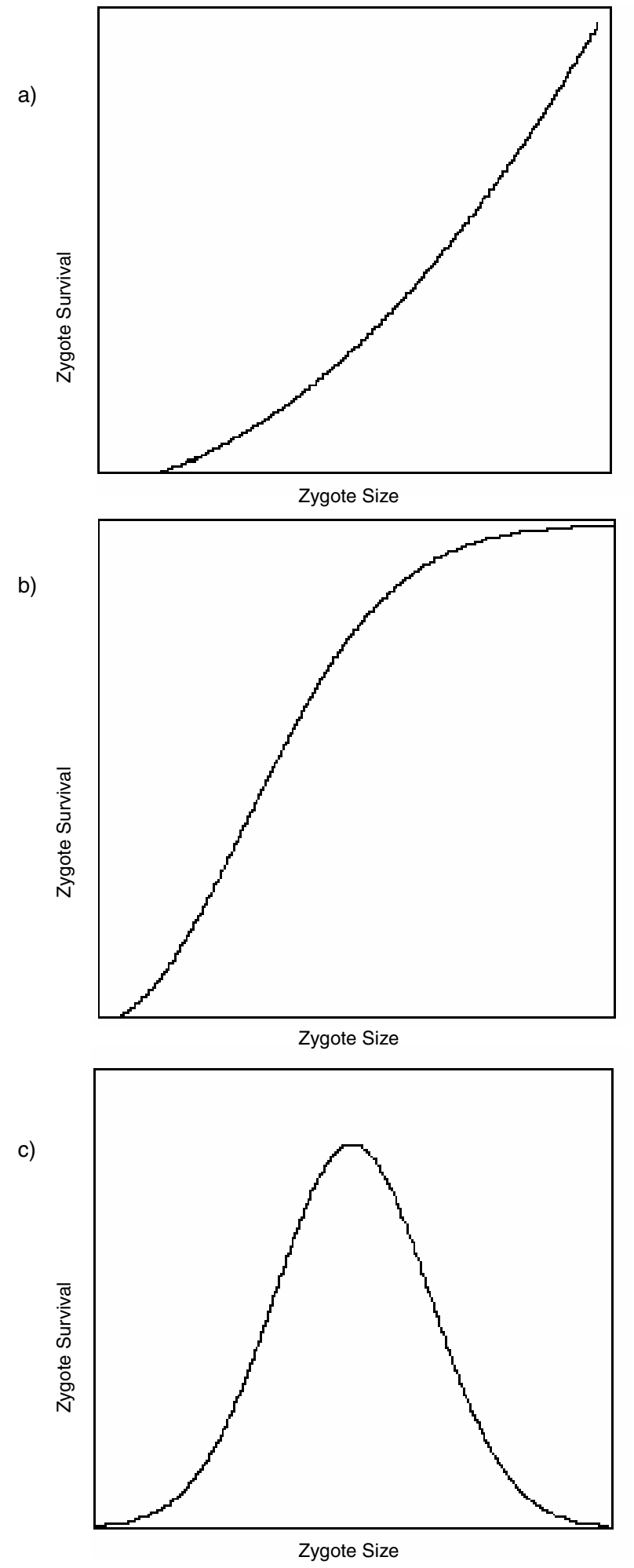

FIG. 1. General shape of the functions relating zygote size to zygote survival used in the analysis. (a) $K(Z)=a+b Z+c Z^{2}$. Zygote survival is an increasing function of size. (b) $K(Z)=c$ $\exp \left(-Z^{2}\right)$. Zygote survival increases monotonically with size, but approaches a maximum. (c) $K(Z)=\exp \left(-\left(Z-Z_{0}\right)^{2} / 2 \sigma^{2}\right)$. There is an intermediate zygote size maximizing zygote survival, and survival decreases for smaller or larger zygotes

$$
K(Z)=a+b Z+c Z^{2} .
$$

We investigate the existence of evolutionary branching points in dependence of the parameters $a, b$ and $c$. Note that, depending on the choice of these parameters, eq. (14) can produce negative survival values which are not meaningful in the present context. For simplicity, we ignore the sign of the survival function during the investigation of the evolutionary dynamics of the system up to the point where we identify potential branching points. We will then determine whether the gamete sizes corresponding to these potential branching points lead to zygotes with a positive survival probability.

Equation (10) yields

$$
x^{*}=\frac{-a}{b}
$$

as the singular point, i.e. as that point in allele space at which the selection gradient vanishes. Thus, as long as $a$ and $b$ in eq. (14) are of opposite signs there is a biologically feasible singular point at $x=-a / b>0$. Equation (11) yields

$$
\frac{2 c}{-a+\frac{4 c a^{2}}{b^{2}}}<\frac{b^{2}}{2 a^{2}}
$$

as the condition for the singular point $x^{*}$ to be an evolutionary attractor. Since the denominator on the left-hand side of eq. (16) is equal to $K\left(2 x^{*}\right)$ and therefore positive, this conditions translates into

$$
a b^{2}<0
$$

Since $b^{2}>0$ for all $b$, this shows that the singular point given by (15) is an evolutionary attractor if and only if $a<0$. This in turn shows that for any $a<0$ and $b>0$ there exist singular strategies that are evolutionary attractors. On the other hand, if $a>0$ and $b<0$ the singular point $x^{*}=-a / b$ is still biologically feasible, but it is a repellor for the adaptive dynamics. In this case gamete size would evolve to ever smaller values if the initial gamete size is smaller than $x^{*}$, and it would evolve to ever larger values if the initial gamete size is larger than $x^{*}$. Thus, with $a>0$ and $b<0$ the adaptive dynamics depend on the initial conditions, and the evolution of gamete size would only come to a halt due to external constraints that are not part of the model description. 
Pursuing the investigation for cases with $a<0$ and $b>0$, for which $x^{*}=-a / b$ is an attractor, we see from eq. (13) that the condition for these singular strategies to be evolutionarily unstable, and hence to be the starting point for evolutionary branching and possibly the evolution of anisogamy, is simply that

$$
c>0 \text {. }
$$

Equations (15)-(18) show that with zygote survival $K(z)$ given by (14), the adaptive dynamics of gamete size have an evolutionary branching point if $a<0, b>0$ and $c>0$. A population that is at this branching point will produce gametes of size $-a / b$ that will fuse to form zygotes of size $-2 a / b$. Inserting this zygote size into eq. (14) leads to the survival probability of these zygotes:

$$
\begin{aligned}
K\left(-2 \cdot \frac{a}{b}\right)= & a+b \cdot\left(-2 \cdot \frac{a}{b}\right)+c \cdot\left(-2 \frac{a}{b}\right)^{2}= \\
& =-a+c \cdot\left(-2 \frac{a}{b}\right)^{2} .
\end{aligned}
$$

As $a$ is negative and $c$ is positive, $K(-2 a / b)$ is positive irrespective of the exact value of $a$ and $c$. This indicates that the branching point $(-a / b)$ is associated with a positive zygote survival and therefore biologically meaningful.

To summarize, we find the following dynamic behaviour for $K(Z)=a+b Z+c Z^{2}$. For $a<0$ and $b>0$ the adaptive dynamics has an equilibrium attractor in allele space. The evolutionary stability of this attractor depends on the parameter $c$. If $c<0$, then the attractor is evolutionarily stable and hence the endpoint of the adaptive dynamics. However, if the size-dependent survival function of the zygote is accelerating, so that $c>0$, then the attracting intermediate gamete size is evolutionarily unstable and gives rise to evolutionary branching in allele space and potentially the evolution of anisogamy. This corroborates the finding of Parker that for anisogamy to evolve the benefit (in terms of survival) for larger zygotes has to be greater than proportional to zygote size.

We note, however, that the zygote survival functions $K(Z)=Z^{n}(n>0)$ used in Parker et al. (1972) would not lead to evolutionary branching. In fact, it is easy to see that for $n \neq 2$ the adaptive dynamics generated by these fitness functions do not even have singular points (for $n=2$ every point in allele space would be singular, i.e. the fitness gradient is identically 0 in the whole allele space). In other words, the selection gradients corresponding to these fitness functions are nowhere 0 , and instead these gradients are always positive (for $n>2$ ) or always negative (for $1<n<2$ ). With small mutations, selection gradients that are always positive induce the evolution of ever larger gamete sizes, and selection gradients that are always negative lead to ever smaller gamete sizes. Therefore, with these zygote survival functions one would expect directional evolution if mutations are small, and divergence in gamete size is only possible if the effect of mutations exceeds a critical value. Since selection gradients strictly speaking only predict selection pressures for very small mutations, it is in principle possible that even with a positive selection gradient favouring larger gamete sizes mutations coding for gametes that are significantly smaller than the resident are selectively advantageous. This is exactly what happens in the models of Parker et al. (1972), in which the selection gradient is positive but smaller gametes that are sufficiently different from the resident can also invade, which leads to a dimorphism. The relationship between the exponent $n$ and the minimal effect of mutations required for the evolution of anisogamy in the models of Parker et al. (1972) has been described by Charlesworth (1978). For studying gradual evolutionary diversification in gamete size due to arbitrarily small mutations, however, these zygote survival functions do not seem feasible.

In order to emphasize the generality of our approach, we now apply our analysis to other zygote survival functions. As Parker et al. pointed out a zygote survival function that is an increasing and accelerating function of size is probably not very realistic. As this is a requirement for the evolution of anisogamy when zygote fitness is a quadratic function as in eq. (11) [see Parker et al. (1972), and previous paragraphs] we investigated whether evolutionary branching also occurs for more realistic survival functions. Such a function is

$$
K(Z)=c-\exp \left(-Z^{2}\right)
$$

which has a sigmoid shape: fitness increases monotonically with size, but approaches a maxi- 
mum (Fig 1b). By repeating the steps of finding singular points, checking whether they are attractors for the adaptive dynamics, and investigating their evolutionary stability, we find that the behaviour of the system based on the function (20) is similar to the previous case. More specifically, for $0<c<1$, we find that a singular strategy exists. This singular strategy is always an attractor for the adaptive dynamics. If $c>0.5$, the singular point is a fitness minimum and hence an evolutionary branching point, i.e. the starting point for the evolution of anisogamy. If $c<0.5$, then the singular point is an ESS, representing the evolutionary stable gamete size of a population with isogamy. Thus, with the survival function (20) the evolution of anisogamy again occurs for a whole range of parameters and is therefore a robust and generic feature of the model.

Finally, we consider the survival function

$$
K(Z)=\exp \left(-\frac{\left(Z-Z_{0}\right)^{2}}{2 \sigma^{2}}\right)
$$

which describes a situation in which there is an optimal zygote size $Z_{0}$, and zygote survival decreases for smaller or larger zygotes, with the rate of decrease described by the parameter $\sigma$ (Fig. 1c). Analysis of eqs (9)-(13) reveals that this function leads to qualitatively different behaviour of the adaptive dynamics. For $Z_{0}^{2}<8 \sigma^{2}$ there is no singular strategy, and gamete size decreases over time. For $Z_{0}^{2}>8 \sigma^{2}$ there are two different singular strategies. The lower one, situated at $x_{1}^{*}=$ $\left(Z_{0}-\sqrt{Z_{0}^{2}-8 \sigma^{2}}\right) / 4$, is a repellor for the adaptive dynamics. Thus, if gamete size in the initial population is smaller than $x_{1}^{*}$ it evolves towards zero. If the initial gamete size is larger than $x_{1}^{*}$, then gamete size increases towards the upper singular strategy $x_{2}^{*}=\left(Z_{0}-\sqrt{Z_{0}^{2}-8 \sigma^{2}}\right) / 4$, which is always an attractor for the adaptive dynamics. This attracting singular point can again be either a fitness minimum and hence a branching point, which happens if $Z_{0}^{2}<9 \sigma^{2}$, or it can be a fitness maximum and hence an ESS, which occurs for $Z_{0}^{2}>9 \sigma^{2}$. Thus, with the survival function (21) the behaviour of the system changes qualitatively: there are either two or no singular points, and whether or not the system undergoes evolutionary branching may depend on the initial gamete size in the population. Nevertheless, it is again true that evolutionary branching occurs for a range of parameter values.

Overall, we have thus seen analytically that the formation of two clusters in allele space can occur generically for a number of qualitatively different functions of zygote survival even if mutations are arbitrarily small. In the next section we will investigate the evolutionary dynamics occurring subsequent to evolutionary branching using numerical simulations.

\section{Individual based simulations}

The analytical theory of the preceding section relies on some simplifying assumptions. Most importantly, our invasion function assumes a mutant allele to occur only in heterozygous individuals. However, if the allele is successful when rare it is implicitly assumed that, if the fitness gradient is non-zero, it will not only invade but subsequently oust the previous resident and become the new resident. Obviously, during this substitution the assumption that the new gamete allele only occurs in heterozygotes will be violated. Furthermore, adaptive dynamics views evolution as a process that is limited by the rate of mutations. A population evolves by the sequential invasion of successful mutants. This greatly facilitates the analysis, but is clearly unrealistic. Rather, in a setting in which most mutations are of small effect a certain degree of polymorphism is expected to be maintained by mutation-selection balance. In addition, even if the populations do converge to fitness minima, making it possible for alleles coding for different gamete sizes (on either side of the fitness minimum) to coexist, the genetic assumptions of our model will lead to the formation of three different zygote genotypes resulting from the coexistence of two different gamete size alleles. Therefore, it is not clear a priori which zygote types will eventually emerge from the evolutionary branching process. For these reasons, and even though it is in principle possible to study evolution in polymorphic populations using the analytical tools of adaptive dynamics, we used individual-based computer simulations to investigate the evolution of gamete size in populations that become polymorphic through evolutionary branching. 
For our simulations we used a model population with a constant population size of 1,000 individuals with discrete generations. As before, these diploid individuals reproduce by producing haploid gametes of a size determined by the parental genotype. Each gamete carries one of the parental size determining alleles. Fusions between gametes occur randomly and yield zygotes of a size that equals the sum of the sizes of the two fusing gametes. The probability of a newly formed zygote to survive to reproduce is size dependent. We use the following procedure to follow the genetic composition of the population through time: We first determine all possible matings (i.e. all possible fusions of gametes) in a given generation based on the genotypes that are present. We then calculate the relative frequencies of matings and multiply these by the survival probabilities of the resulting zygotes. These products are proportional to the probabilities that the corresponding zygotes will be formed and survive to reproduction. Therefore, normalizing these quantities (by adjusting their sum to 1) and drawing the 1,000 individuals of the next generation randomly according to the normalized products completes one generation in the individual-based model.

The starting population is monomorphic, and the evolutionary process is driven by occasional mutations. Mutations occur during gamete production with a rate $\mu=10^{-2}$ per reproductive event. The effect of a mutation is normally distributed with mean of zero and standard deviation $\sigma=q \cdot x_{0}$, where $q$ is a small constant and $x_{0}$ is the original trait value. The assumption that mutations have a standard deviation proportional to the parental trait value prevents the occurrence of unrealistically large mutations when the original trait value is very small, and it appropriately scales the speed of evolution. We limit the range of possible values for gamete size determining alleles between 0.1 and 200 units on an arbitrary scale. All simulations were run until the phenotype distribution reached a stable state.

We first consider zygote survival function $K(Z)=a+b Z+c Z^{2}$, eq. (14). If parameters are chosen within the range demonstrated in the previous section to lead to evolutionary branching, gamete size evolves to the singular strategy regardless of the starting conditions. At this point mutant alleles coding for both smaller and larger sizes invade, leading to evolutionary branching in allele space. However, as the two branches in allele space emerge, there are three corresponding branches in diploid genotype space (Fig. 2). In other words, the two emerging clusters of gamete size alleles pro duce three clusters of zygote genotypes, representing the two homozygotes and the heterozygote of the two allelic branches. These two allelic branches
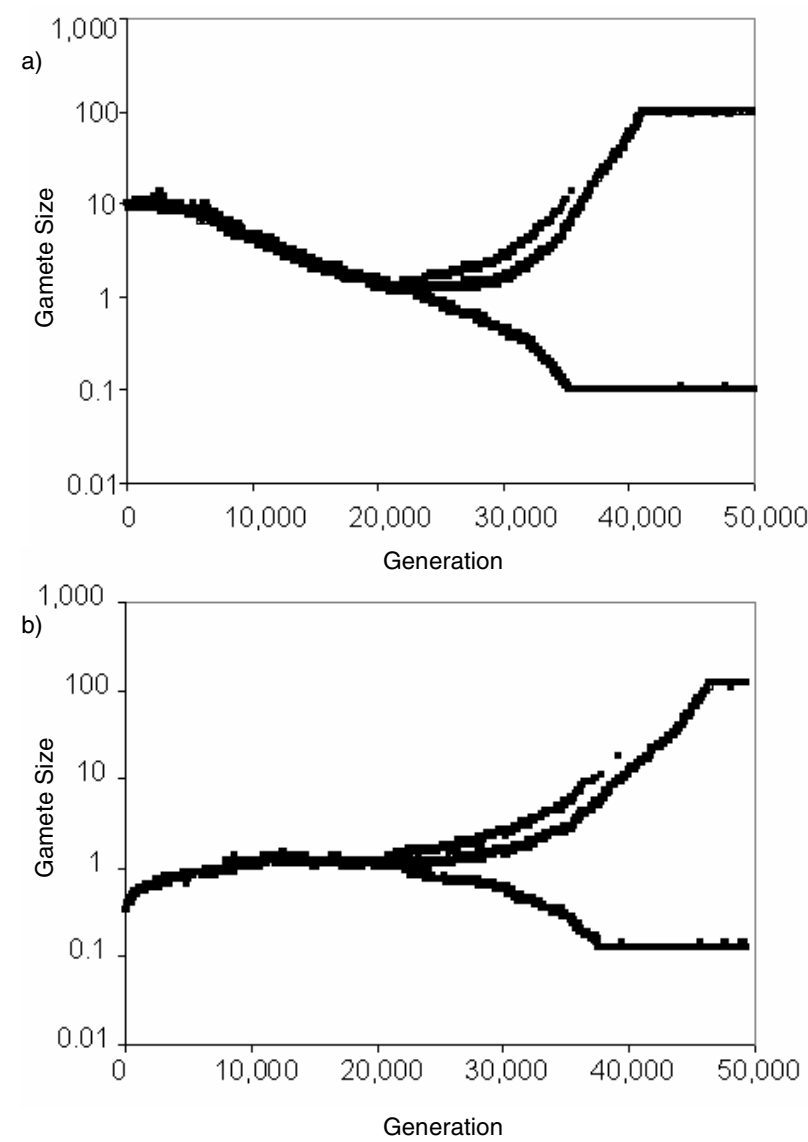

FIG. 2. Individual-based simulations of the evolution of gamete size if zygote survival is an increasing function of zygote size (cf. Fig. 1a). The dots represent zygotes that produce gametes falling in a certain size class (a dot stands for a size class that involves at least two zygotes after size-dependent mortality has been imposed). Irrespective of the starting point [compare (a) to (b)] gamete size evolves to an intermediate value where both alleles coding for smaller gametes and for larger gametes invade. This results in the formation of three types of zygotes that differ in the size of the gametes they produce. The zygote type that produces large gametes becomes rare and disappears, leading to anisogamy with two types of individuals that differ in the size of the gametes they produce. Initial allele size: (a) $X=10$ and (b) $X=0.3$. Zygote survival function: $K(Z)=-1+Z+Z^{2}$. Population size: 1,000 . Mutations occur at a rate $\mu=10^{-2}$ with a standard deviation determined by $q=0.02$ (see text for further details) 
diverge until they reach the boundaries of the specified range of gamete size (Fig. 2). Once the two allelic branches diverged enough, matings producing viable zygotes occur exclusively between gametes of individuals producing small gametes (homozygous for the small allele) and those producing intermediate sized gametes (heterozygotes). This is because small gametes become so numerous that they are involved in almost all fusions. However, zygotes formed from two small gametes are too small to be viable. Therefore, with a certain degree of divergence between the two alleles, only two diploid zygote genotypes persist, and both are formed as intermediate sized zygotes. The first of these zygote genotypes is homozygous for the small allele and produces small gametes that are genetically uniform. The second zygote genotype is heterozygous and produces gametes of intermediate size that either carry an allele coding for small or for large gametes. Overall, this scenario therefore corresponds to the evolution of anisogamy. Simulations using the sigmoid zygote survival function (20) show a similar outcome. However, in contrast to the previous scenario, the upper branch does not evolve towards the upper limit of gamete size, because the advantage of larger zygotes decreases with increasing size. The branches therefore do not diverge very much and as a result all three diploid genotypes created by the two alleles are present in the population. An example of this is shown in Figure 3a. Each of the three genotypes produces gametes of a characteristic size. Whereas each of the two homozygous genotypes produces gametes that are genetically uniform, the heterozygous genotype produces gametes of intermediate size that carry an allele coding either for small or large gametes. There are thus four different types of gametes present when the population reaches a stable state.

If the lower limit for gamete size is decreased, the genotype carrying two large alleles disappears, because small gametes become so numerous that they are involved in all zygote formations. The stable state is then similar to what is observed with zygote survival function (14) in Figure 2, except that, as mentioned, the large allele does not reach the upper limit of gamete size. An example of this scenario is shown in Figure $3 b$.

With sigmoid relationships between the size of a zygote and its survival the evolution of anisog-
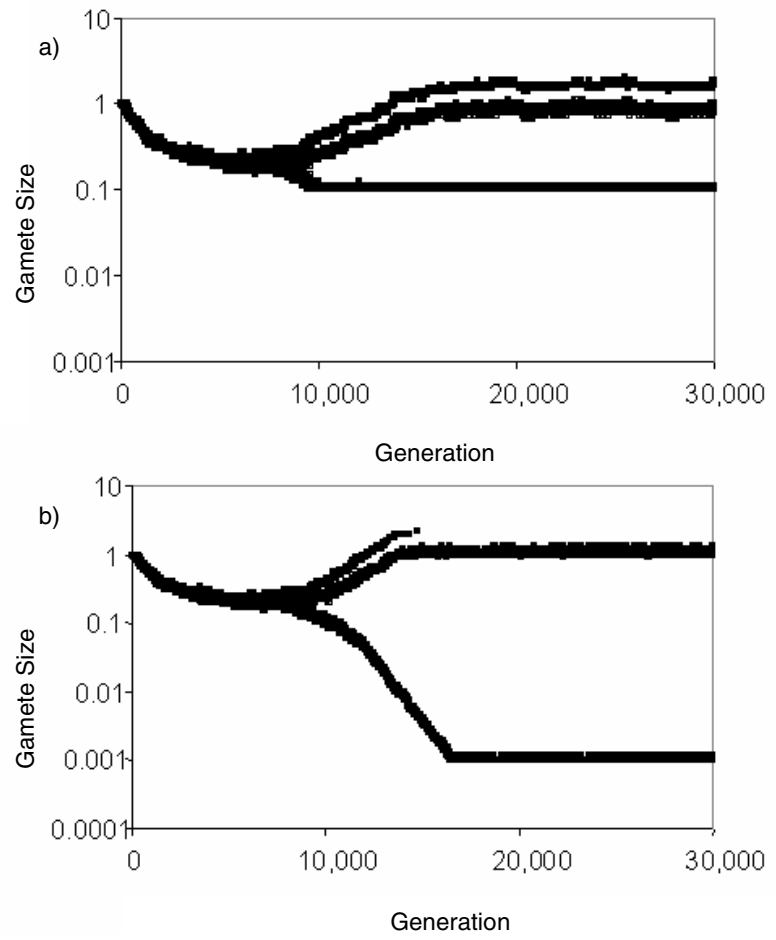

FIG. 3. Individual-based simulations of the evolution of gamete size if zygote survival is a sigmoid function of zygote size (cf. Fig. 1b). The dots represent zygotes that produce gametes falling in a certain size class (a dot stands for a size class that involves at least two zygotes after size-dependent mortality has been imposed). (a) If the lower size limit for the gametes is rather high (lower limit $=0.1$; see text for details), three diploid zygote genotypes that differ in the size of the gametes they produce coexist when a stable state is reached. (b) If the lower size limit for the gametes is low (lower limit $=0.001$ ) then the zygote type that produces large gametes becomes rare and disappears, leading to an anisogamous population consisting of two types of individuals that differ in the size of gametes they produce. Initial allele size: $X=1$. Zygote survival function: $K(Z)=\mathrm{c}-\exp \left(-Z^{2}\right)$ with $c=0.99$. Population size: 1,000 . Mutations occur at a rate $\mu=10^{-2}$ with a standard deviation determined by $q=0.03$

amy therefore depends on biological details, namely on how small gametes can be without loosing functionality. The smaller they can be the more numerous they become and hence the more likely is the evolution of anisogamy.

As predicted by the analytical theory, the Gaussian zygote survival function (21) produces a different pattern: with Gaussian zygote survival the evolutionary dynamics of the individual-based model typically depend on the initial conditions. For parameter settings for which two singular strategies exist, the singular point with the lower gamete size is an evolutionary repellor, and gamete size 
evolves towards the lower limit set in the simulation if the initial gamete size is smaller than this repellor. If the initial gamete size is larger than the repellor, the population evolves to the upper singular strategy. If this singular point represents a branching point, the population splits into initially three distinct genotypic branches. However, as they diverge further, the two upper branches, and therefore the allele coding for large gamete size, go extinct, and the lower branch evolves to ever smaller gamete sizes. The reasons for this evolutionary pattern are that the selective disadvantage of producing only few (but large) gametes is initially balanced by the higher survival probability of the resulting zygotes. However, similar to the previous example, the survival advantage of larger gametes decreases with increasing size, as the size of the larger gametes approaches the optimal zygote size determined by the Gaussian survival function $K(z)$. Moreover, with a Gaussian survival function there is no lower size limit a zygote must exceed to be viable (because zygote survival is always positive even if the size of the fusing gam-

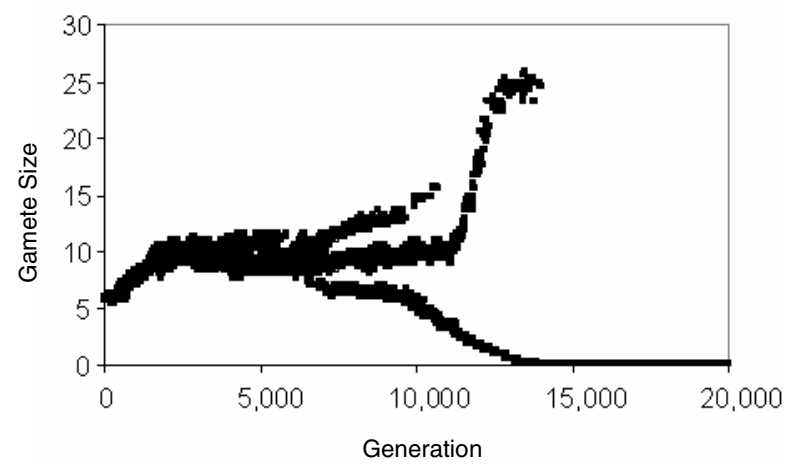

FIG. 4. Individual-based simulations of the evolution of gamete size if zygote survival reaches a maximum at an intermediate zygote size and decreases for larger and smaller zygotes (cf. Fig. 1c). The dots represent zygotes that produce gametes falling in a certain size class (a dot stands for a size class that involves at least two zygotes after size-dependent mortality has been imposed). A population that is initiated in the vicinity of the branching point evolves to this branching point where it splits into three branches representing three zygote types differing in the size of the gametes they produce. The zygotes producing gametes of large and intermediate size become rare and disappear, whilst the population has left the basin of attraction of the branching point and evolves to the lower size limit. Initial allele size: $X=6$. Zygote survival function: $K(Z)=\exp \left(-\left(Z_{0}-Z\right)^{2} / 2 \sigma^{2}\right)$ with $Z_{0}=0.29$ and $\sigma=10$. Population size: 1,000 . Mutations occur at a rate $\mu=10^{-2}$ with a standard deviation determined by $q=0.03$ exceed to be viable (because zygote survival is always positive even if the size of the fusing gametes approaches zero), and even gametes of the minimal size allowed in the model can fuse to become viable zygotes (although with a low survival probability). As a consequence of the evolution of ever smaller gametes, and hence of ever bigger numbers, in one allelic branch, the other branch consisting of the large gametes goes extinct. The lower branch meanwhile has left the basin of attraction of the branching point, i.e. it has evolved to be smaller than the lower singular point, and subsequently evolves towards the lower end of the range of gamete size. Thus the population evolves to the lower limit of gamete size.

This scenario is illustrated in Figure 4 and is typical of the adaptive dynamics when Gaussian zygote survival is such that an evolutionary branching point exists.

In particular, with Gaussian zygote survival a gamete size polymorphism can only be maintained if a lower limit for gamete size that is substantially bigger than 0 is imposed, for only in that case are the large alleles not swamped by the small alleles. However, increasing the lower limit for gamete size generally implies that at the evolutionary endpoint three genotypes coexist that produce four types of gametes, similar to the outcome shown in Figure 3a.

\section{Discussion}

Anisogamy, the production of gametes of two different sizes, is a derived state that evolved from isogamy several times independently (Vorontsov, 1973). It is very widespread among sexually reproducing organisms, an observation that asks for a general explanation for its evolution. The explanations for the evolution of anisogamy that have been put forward so far either required mutations with large effects (e.g. Parker et al., 1972; Charlesworth, 1978; Maynard Smith, 1978), or they assumed that anisogamy evolved in populations that already had mating types (Charlesworth, 1978; Matsuda and Abrams, 1999). Whereas both assumptions are biologically realistic, it is certainly worthwhile to analyse whether they are necessary to explain the evolution of anisogamy. This was the goal of this paper - to investigate whether ani- 
sogamy readily evolves in an isogamous population without mating types under a broad range of conditions and with mutations of arbitrary small effects. As in the previous models, we assume that gamete size is exposed to two opposing forces: Production of a large number of small gametes increases the potential number of fusions, whereas production of a smaller number of larger gametes increases the survival of the resulting zygotes. The evolution of gamete size is then determined by the dependence of zygote survival on zygote size and by the genetics underlying zygote size. To investigate the evolution of gamete size under a broad range of conditions, we consider three different functions relating zygote size to zygote survival. Using these different zygote fitness functions, we investigated the evolutionary dynamics of gamete size using the framework of adaptive dynamics, which is based on studying the fate of rare mutant alleles differing in their effect on the trait under consideration - gamete size in our example - from the pool of resident alleles.

We applied this analysis to all three functions relating zygote size to zygote survival. For all three functions we found ranges of parameters where the allele pool of the population is expected to evolve to a fitness minimum in allele space. (It is interesting to note that for the zygote survival function used in the original model of Parker et al. (1972) no such fitness minima exist, which is reflected by the fact that this model requires mutations whose effect exceeds a critical size (Charlesworth, 1978)). Once the population has converged to such an evolutionary branching point, mutant alleles coding for smaller as well as mutant alleles coding for larger gametes than the resident alleles can invade. Such a situation is expected to lead to the splitting of the allele pool of the population into two distinct clusters, one cluster representing alleles coding for small gametes, the other cluster representing alleles coding for large gametes. Whereas this split of the allele pool is a prerequisite for anisogamy, such a split is not necessarily sufficient for the evolution of anisogamy. Natural anisogamous populations are characterized by the presence of two genotypes that differ in the size of the gametes produced. In contrast, the two clusters in allele space will initially give rise to three different types of genotypes, one composed of two alleles from the first cluster, one composed of two alleles from the second cluster, and one with an allele from each of the two clusters. The phenotypes that are produced by these three types of genotypes depend on dominance. If the alleles act additively, as we assume in our analysis, then each of these three types of genotypes produces gametes of a characteristic size. If either alleles for small gametes or alleles for large gametes are dominant, then the three types of genotypes give rise two types of phenotypes producing large and small gametes, respectively. Whereas this situation phenotypically corresponds to anisogamy, it differs from the natural situation with only two genotypes forming the two sexes. The situation with two genotypes emerges if one of the two homozygotes formed by the two different alleles (or two different types of alleles) is lost. This happens if matings occur exclusively between gametes formed by the other two genotypes. There are two mechanisms that can account for this restriction. The first mechanism involves pre-existing mating types. In a population with mating types anisogamy is expected to evolve through linkage between loci determining the mating type and loci determining gamete size (Matsuda and Abrams, 1999). Matings will then exclusively occur between small gametes of the first mating type and large gametes of the second mating type. The second mechanism does not involve pre-existing mating types and corresponds to the situation analyzed here: If the two clusters in allele space diverge a lot, the small gametes will further decrease in size and therefore become very numerous. This has two effects. First, they will become so small that the fusion of two small gametes will not give rise to a viable zygote. Second, they will become so numerous that they will be involved in all fusions. As a consequence, all the viable zygotes will be formed by the fusion of a small and a large gamete. This process can be reinforced by the evolution of non-random fusion of gametes: In a population where gametes of unequal size fuse randomly alleles inducing disassortative fusion of gametes can spread and go to fixation (Parker et al., 1972). The evolution of disassortative fusion of gametes leads to a system where fusions exclusively occurs between small and large gametes, which corresponds to the situation found in natural anisogamous species (with only few known exceptions, e.g. the volvoid Pandorina (Parker et al., 1972)). 
These evolutionary changes, occurring after the split of the allele pool, are a crucial aspect of the evolution of anisogamy, which we have investigated using individual-based computer models. The results of these simulations corroborate and extend the analytical results: The conditions for which the analysis predicts that the allele pool will evolve to a branching point indeed leads to the formation of two clusters of alleles in the individual-based computer model. These two clusters in allele space will initially give rise to three types of genotypes. The fate of these genotypes then depends on the function relating zygote size to zygote survival. For the first two zygote survival functions, we find ranges of conditions where one of the genotypes disappears. This scenario corresponds to the evolution of anisogamy with two genotypes differing in the size of the gametes they produce, so that the heterogametic genotype produces large gametes and the homogametic genotype produces small gametes. For the third function, dimorphism of alleles determining gamete size is either transient or leads to a system with more than two genotypes, which does not correspond to the situation in natural populations.

In summary, we investigated the evolution of gamete size through mutations of small effect in a population without mating types and under different assumptions about how zygote survival depends on zygote size. The evidence for this scenario leading to the evolution of anisogamy is mixed. For all functions relating zygote size to zygote survival we find emergence of a dimorphism through evolutionary branching in the alleles determining gamete size under suitable conditions. Whether this dimorphism will give rise two genotypes that differ in the size of the gametes they produce depends on the details of how zygote survival depends on zygote size. Whether the models investigated here describe realistic scenarios for the evolution of anisogamy therefore depends on how zygote survival depends on zygote size in natural populations.

It is interesting to note that this basic ingredient, i.e. the function relating size to survival, has a classical application in the analysis of the evolution of seed size. In these models there is also a tradeoff between size and number of seeds, so that the fitness of a particular seed size phenotype $\mathrm{s}$ is proportional to $K(s) / s$, where $K(s)$ is survival as a function of size. This is very similar to our eq. (1), which describes the reproductive output in populations that are monomorphic for a given gamete size allele. However, in their simplest form seed size models do not incorporate frequency-dependence and therefore always lead to an intermediate seed size ESS see Geritz et al. (1999), where various extensions of the basic model are discussed which do indeed lead to a number of more interesting scenarios for the evolutionary dynamics of seed size). In contrast, and even though we use the same basic fitness function and without further assumptions about factors influencing reproductive success, our models for gamete size include frequency-dependence, essentially because of the genetic details, i.e. because the fitness of a gamete depends on its size as well as on the size of the other gamete with which it fuses to form a zygote.

That these models lead to evolutionary branching represents a nice twist to the often heard objection against evolutionary branching that this phenomenon only occurs in asexual models. In our analysis the mere inclusion of genetic detail into an otherwise dynamically uninteresting model creates the potential for evolutionary branching (see e.g. Dieckmann and Doebeli, 1999; or Kisdi and Geritz, 1999, for general approaches to study evolutionary branching in sexual populations).

In sum, evolutionary branching in alleles coding for gamete size represents a theoretically plausible scenario for the evolution of anisogamy and thus an alternative to the scenarios in which the evolution of mating types is assumed to have preceded the evolution of anisogamy. Once mating types have evolved isogamy is unstable under the assumptions of size dependent zygote survival and invariant reproductive effort (Matsuda and $\mathrm{Ab}$ rams, 1999). Our models do of course not answer the question of whether, in any given case, anisogamy evolved before or after the establishment of different mating types. In fact, isogamous species without mating types seem to be rare (Hoekstra, 1987; Maynard Smith and Szathmary, 1995), which indicates that the evolution of mating types may have preceded the evolution of anisogamy. On the other hand, once evolved anisogamy would be likely to favour the evolution of mating types in a process akin to reinforcement. Therefore it is difficult to infer the sequence of evolutionary events from the co-occurrence of mating types and ani- 
sogamy. Our models show that, at least in principle, mating types are not a prerequisite for the evolution of anisogamy.

\section{References}

Bell, G. (1978): The evolution of anisogamy. J. Theor. Biol. 73:247-270.

Bell, G. (1982): The Masterpiece of Nature. Croom Helm Ltd., London.

Bulmer, M. (1994): Theoretical Evolutionary Ecology. Sinauer, Sunderland, MA.

Charlesworth, B. (1978): The population genetics of anisogamy. J. Theor. Biol. 73:347-357.

DIECKMANN, U. and LAW, R. (1996): The dynamical theory of coevolution: A derivation from stochastic processes. $J$. Math. Biol. 34:579-612.

Dieckmann, U. and Doebeli, M. (1999): On the origin of species by sympatric speciation. Nature 400:354-357.

Geritz, S. A. H., Kisdi, E., Meszena, G., Metz, J. A. J. (1998): Evolutionary singular strategies and the adaptive growth and branching of the evolutionary tree. Evol. Ecol. 12:35-57.

Geritz, S. A. H., van der Meijden, E. and Metz, J. A. J.
(1999): Evolutionary dynamics of seed size and seedling competitive ability. Theor. Pop. Biol. 55:324-343.

Kisdi, E. and Geritz, S. A. H. (1999): Adaptive dynamics in allele space: Evolution of genetic polymorphism by small mutations in a heterogeneous environment. Evolution 53:993-1008.

HoEKSTRA, R. F. (1980): Why do organisms produce gametes of only two different sizes? Some theoretical aspects of the evolution of anisogamy. J. Theor. Biol. 87:785-793.

HoEkstra, R. F. (1987): The evolution of sexes. In STEARnS, S. C. (ed.): The Evolution of Sex and its Consequences. Birkhäuser, Basel, pp. 59-91.

MAtSUDA, H. and ABRAMS, P. A. (1999): Why are equally sized gametes so rare? The instability of isogamy and the cost of anisogamy. Evol. Ecol. Res. 1:769-784.

MAYNARD SMith, J. (1978): The Evolution of Sex. Cambridge University Press, Cambridge.

Maynard Smith, J. and Szathmary, E. (1995): The Major Transitions in Evolution. W. J. Freeman, San Francisco.

PARKer, G. A., BAKer, R. P. and SMith, V. G. F. (1972): The origin and evolution of gamete dimorphism and the malefemale phenomenon. J. Theor. Biol. 36:529-553.

VORONTSOV, N. N. (1973): The evolution of the sex chromosomes. In Chiraelli, A. B. and Capanna, E. (eds): Cytotaxonomy and Vertebrate Evolution. Academic Press, New York, $646 \mathrm{pp}$. 
